Available online at GSC Online Press Directory

GSC Biological and Pharmaceutical Sciences

e-ISSN: 2581-3250, CODEN (USA): GBPSC2

Journal homepage: https://www.gsconlinepress.com/journals/gscbps

(RESEARCH ARTICLE)

\title{
Evaluation of acute pain management in ambulatory herniorrhaphy during free surgical outreach in Akwa Ibom State, South-South Nigeria.
}

\author{
Etta Otu Enenyi ${ }^{1}$, Ekpe Eyo ${ }^{2}$, Inyang Udoinyang Clement ${ }^{3}$, Udeme Nsese ${ }^{1}$ and Ekpenyong Christopher Edet \\ $4, *$
}

${ }^{1}$ Department of Anaesthesia, University of Uyo Teaching Hospital, Uyo, Akwa Ibom State.

2 Department of Surgery, University of Uyo Teaching Hospital, Uyo, Akwa Ibom State.

${ }^{3}$ Department of Orthopaedics and Traumatology, University of Uyo Teaching Hospital, Uyo, Akwa Ibom State.

${ }^{4}$ Department of Physiology, Faculty of Basic Medical Sciences, University of Uyo, Uyo, Nigeria.

Publication history: Received on 01 May 2020; revised on 09 May 2020; accepted on 11 May 2020

Article DOI: https://doi.org/10.30574/gscbps.2020.11.2.0122

\begin{abstract}
Surgical outreach Program is a platform where a large number of elective surgical patients are treated within a short time, usually on ambulatory basis by a volunteer surgical team at no cost to the patients. Inguinal hernia repair is usually the commonest procedure performed. However, acute postoperative pain associated with it is not often investigated in our environment.

All adult patients with uncomplicated inguinal hernia who consented to the study were recruited. The choice of anaesthesia, surgical technique and postoperative analgesics used were as per the discretion of the individual surgical team members. Postoperatively, the patients were interviewed through a telephone call using a structured questionnaire for three consecutive days after the surgery. Data obtained were analyzed using SPSS version 16.

A total of 43 patients, 34 males, 9 females were recruited. The mean age was 42.86 years. Local anaesthesia (LA) only was the commonest anaesthetic technique (42.2\%), followed by LA + sedation (30.2\%). Unimodal analgesic regimen as against multimodal regimen was used in $46.5 \%$ and $53.5 \%$ patients respectively. The incidence of moderate to severe pain was highest on the first day after surgery (72.5\%), and it was statistically significant compared with No pain/mild pain $(27.5 \%)$, $\mathrm{p}$ value $=0.002$. More patients treated with unimodal compared with multimodal analgesic regimen had moderate to severe pain (56.7\% vs $43.3 \%)$.
\end{abstract}

Surgical outreach program is useful in reducing surgical disease burden. However, the incidence of acute moderate to severe postoperative pain is high (72.5\%). Therefore, a standard protocol that is effectively communicated to all members of the surgical team must be developed in order to reduce this morbidity.

Keywords: Acute postoperative pain; Ambulatory herniorrhaphy; Free surgical outreach

\section{Introduction}

The burden of surgical diseases in rural ccommunities in Sub-saharan Africa is enormous [1, 2]. An estimated one third to one half of the world population lacks basic Surgical Care. In a previous survey in East Africa, only $11 \%$ of women requiring caesarean section got it, only $14 \%$ of patients with inguinal hernia were surgically treated, while $13 \%$ of patients with strangulated hernia were operated upon, accounting for a mortality of about $90 \%[3,4]$.

\footnotetext{
* Corresponding author: Ekpenyong Christopher Edet
} 
This limited surgical access has been attributed to several factors including inadequate human resources, limited surgical supplies, high surgical fees, and poverty $[5,6,7]$. In order to improve access to surgical services in Nigeria, particularly in Akwa Ibom State, surgical camps or outreaches are frequently organised where volunteer surgical teams congregate at a specified place and perform a wide range of mostly elective procedures over a short period at no cost to the Patients $[4,8,9]$.

Previous studies have documented that ambulatory inguinal hernia repair is the commonest procedure performed during surgical outreaches $[5,8,9]$, however, the postoperative pain associated with this procedure is not often investigated $[5,8,9]$. Therefore, the purpose of this study was to evaluate the postoperative acute pain management in ambulatory herniorrhaphy during free surgical outreaches.

\section{Patients and Methods}

This was a prospective observational study of all patients who underwent inguinal hernia repair in three rural communities in Akwa Ibom State between May and June 2017 during free surgical outreaches. The facilities included two general hospitals and a newly established medical centre. The two general hospitals had operating rooms and wards which served both as postoperative recovery area and for admission if required, whereas, the newly established medical centre had no dedicated theatre. One large room was improvised to serve both as theatre and recovery area. Ward facility was not available. Patients' follow-up was conducted by the resident health medical officers at the host medical facilities.

Approval for the study was obtained from the local Research Ethical Committees of the host medical facilities. All adults patients scheduled for elective inguinal herniorrhaphy on a day-stay basis from whom an informed verbal consent was obtained were recruited into the study. The exclusion criteria included children from 0-17 years, patients with other pathologies apart from uncomplicated inguinal hernias only and those requiring ward admission. Also, those without functional telephones were equally excluded.

All surgeries were performed by either specialist surgeons or medical officers, while anaesthesia was provided by physician anaesthetists, nurse anaesthetists or the surgeons. The methods of anaesthesia and postoperative drugs including analgesics were at the discretion of the anaesthesia providers and surgeons. All surgical patients were reviewed by the medical officers in the clinic and sent to the laboratory for basic investigations which included packed cell volume (PCV), urinalysis and human immune deficiency virus (HIV) serology before they were posted to the theatre for surgery. A pre anaesthetic check was done in the theatre by the anaesthesia providers, after which an Intravenous cannulation was done on the upper limb and $500 \mathrm{mls}$ of $0.9 \%$ normal set up to run at 20-40 drops per minute. All patients were monitored for blood pressure and oxygen saturation. Spinal anaesthesia was performed using $10 \mathrm{mg}$ of heavy bupivacaine, total intravenous anesthesia (TIVA) was done using a combination of ketamine (80-120 mg), diazepam (5-10 mg) and pentazocine (10-30 mg), while sedation was done with ketamine (10-20 mg), diazepam (3-5 $\mathrm{mg}$ ) and pentazocine (10 mg), all patients except one who had spinal anaesthesia received local anaesthesia (LA) with 20-40 mls of 1\% lignocaine with adrenaline. Oxygen in a cylinder and other resuscitation equipments were available in the theatre for use in event of any critical incident.

Postoperatively, patients were monitored for vital signs in the designated recovery areas and discharged home with appropriate drugs (paracetamol tablet 1 gram three times daily; Ibuprofen tablet $400 \mathrm{mg}$ two times, and diclofenac tablet $100 \mathrm{mg}$ two times daily in different combination for at least three days) within $24 \mathrm{hrs}$ after surgery. All patients were interviewed in the recovery area before discharge and subsequently at home daily for three consecutive days through a telephone contact using a structured questionnaire about their activity level (No activity = able to use the toilet only; partial activity = able to use the toilet and walk around the immediate environment; full activity = able to walk beyond the immediate environment), drug compliance (full = took all analgesics prescribed; partial = took some of the prescribed analgesic; No = took no analgesic prescribed), pain score using the verbal rating scale (graded as: No pain, mild, moderate and severe pain), need for additional analgesics and satisfaction with their pain management using a five point likert scale.

Data in the theatre, recovery area, ward and home were collected into a study proforma. Information obtained included the patient's age, sex, ASA-status and type of hernia. The cadre of surgeon and anaesthesia providers, as well as the postoperative analgesics given were obtained. Data from the telephone interviews were equally obtained. 


\subsection{Date Analysis}

Continuous variables were presented as mean and range, while categorical variables were presented as proportion, frequency and percentage. Difference of proportion was determined using Chi-square, and a p-value $<0.05$ was considered statistically significant. All data analysis was performed using Statistical Package for Social Sciences (SPSS) version 16.

\section{Results}

One hundred and forty eight Patients were surgically treated, 80 (54\%) patients had inguinal hernia. Forty three patients met the inclusion criteria and were recruited for the study. Three patients were lost to follow up on day 1, while 4 were lost on day 2 and 3 because their phones were switched off. Of the 43 patients studied, 34 were males while 9 were females giving a male to female ratio of 3.8:1. The mean age was 43.86yrs with a range of 18-70. Thirty seven patients had unilateral hernia while 6 had bilateral hernia (Table 1).

Table 1 Patients' characteristics

\begin{tabular}{ll}
\hline Category & Number (n) \\
\hline Age (years) & \\
Mean/ & 42.86 \\
Standard deviation & +13.828 \\
Range & $18-70$ \\
Sex: $n(\%)$ & \\
Male & $34(79.1)$ \\
Female & $9(20.9)$ \\
ASA Status n(\%) & \\
ASA I & $31(72.1)$ \\
ASA II & $10(23.3)$ \\
ASA III & $2(4.6)$ \\
Type of Hernia n (\%) & \\
Unilateral & $37(86.0)$ \\
Bilateral & $6(14.0)$ \\
\hline \multicolumn{2}{r}{ ASA- American Society of Anesthesiologists } \\
\end{tabular}

Table 2 shows that majority of the patients were treated by medical officers compared to specialist surgeons (30 vs 13 ). On the other hand, majority (29) of the patients were anaesthetized by physician anaesthetists while 7 each were anaesthetized by nurse anaethelists and surgeons. Local anaethesia (LA) alone was the predominant anaesthetic technique (19), followed by LA + sedation (13), while spinal anaesthesia (SA) was used in one patient only. Majority of the patients were satisfied with their pain management (74.5\%). 
Table 2 Surgical Team Profile, Type of Anaesthesia and Patients' Satisfaction

\begin{tabular}{lll}
\hline Category & Number of Procedures & Percentage (\%) \\
\hline Cadre of Surgeons & 13 & \\
Specialist & 30 & 30.2 \\
Medical Officers & & 69.8 \\
Cadre of Anaesthetists & 29 & \\
Physician & 7 & 67.4 \\
Nurse & 7 & 16.3 \\
Surgeon & & 16.3 \\
Type of Anaesthesia & 2 & \\
TIVA & 19 & 4.7 \\
LA & 8 & 44.2 \\
LA + TIVA & 13 & 18.6 \\
LA + Sedation & 1 & 30.2 \\
Spinal & & 2.3 \\
Patients' Satisfaction & 0 & \\
Very dissatisfied & 5 & 0 \\
Dissatisfied & 6 & 11.6 \\
Neutral & 30 & 14.0 \\
Satisfied & 2 & 69.8 \\
Very Satisfied & TIVA - Total Intravenous Anaesthesia, LA - Local Anaesthesia
\end{tabular}

A combination of paracetamol and Ibuprofen was the most commonly used analgesic regimen (41.9\%), while paracetamol only was the least prescribed medication (2.3\%), Table 3.

Table 3 Postoperative Analgesics prescribed and given to the Patients

\begin{tabular}{llll}
\hline Name of analgesics & Frequency (\%) & Prescriber \\
\hline & & Medical Officer & Specialist Surgeon \\
\hline Paracetamol only & $1(2.3)$ & $1(100)$ & $0(0)$ \\
Ibuproten only & $9(20.9)$ & $6(66.7)$ & $3(33.3)$ \\
Diclofenac only & $10(23.3)$ & $7(70.0)$ & $3(30.0)$ \\
Paracetamol + Ibuproten & $18(4.9)$ & $12(66.7)$ & $6(33.3)$ \\
Paracetamol + Diclofenac & $3(7.0)$ & $3(100)$ & $0(0)$ \\
Paracetamol+Ibuproten+Diclofenac & $2(4.7)$ & $1(50)$ & $1(50)$ \\
Total & $43(100)$ & $30(69.8)$ & $13(302)$ \\
\hline \multicolumn{2}{c}{} \\
\hline
\end{tabular}

Table 4 describes the postoperative recovery characteristics of the patients. Drug compliance was poorest on the day of surgery (69.8\% of the patients did not take their drugs), whereas the compliance remarkably improved after 24 hours. No patient returned to full activity on the day of surgery, however, activity improved progressively throughout the study period, and the patients' satisfaction with their postoperative pain management was rated as high $(69.8 \%$ satisfied and very satisfied). 
Table 4 Postoperative Recovery Characteristics

\begin{tabular}{|c|c|c|c|c|}
\hline Category & At Discharge $(n=43)$ & $\begin{array}{l}\text { Day } 1 \\
(n=40)\end{array}$ & $\begin{array}{l}\text { Day } 2 \\
(n=39)\end{array}$ & $\begin{array}{l}\text { Day } 3 \\
(n=39)\end{array}$ \\
\hline \multicolumn{5}{|c|}{ Drug Compliance n(\%) } \\
\hline Full & $9(20.9)$ & $39(97.5)$ & $39(100)$ & $38(97.4)$ \\
\hline Partial & $4(9.3)$ & $0(0)$ & $0(0)$ & $1(2.6)$ \\
\hline No & $30(69.8)$ & $1(2.5)$ & $0(0)$ & \\
\hline \multicolumn{5}{|l|}{ Activity level (n\%) } \\
\hline Full & $0(0)$ & $0(0)$ & $0(0)$ & $0(0)$ \\
\hline Partial & $11(25.6)$ & $19(47.5)$ & $23(59.0)$ & $33(84.6)$ \\
\hline No & $32(74.4)$ & $21(52.5)$ & $16(41.0)$ & $6(15.4)$ \\
\hline \multicolumn{5}{|c|}{ Extra drug requirement } \\
\hline Yes & $24(55.8)$ & $31(77.5)$ & $23(59.0)$ & $12(30.8)$ \\
\hline No & $19(44.2)$ & $9(22.5)$ & $16(41.0)$ & $27(69.2)$ \\
\hline \multicolumn{5}{|c|}{ Patients' Satisfaction n(\%) } \\
\hline Very dissatisfied & $0(0)$ & $3(7.5)$ & $3(7.7)$ & $2(5.1)$ \\
\hline Dissatisfied & $10(23.3)$ & $8(20.0)$ & $8(20.5)$ & $4(10.3)$ \\
\hline Neutral & $3(7.0)$ & $6(15.0)$ & $8(20.5)$ & $8(20.5)$ \\
\hline Satisfied & $15(34.9)$ & $18(45.0)$ & $19(48.7)$ & $24(61.5)$ \\
\hline Very Satisfied & $15(34.9)$ & $5(12.5)$ & $1(2.6)$ & $1(2.6)$ \\
\hline
\end{tabular}

Majority of the patients experienced moderate to severe pain at discharge and day 1, while moderate to severe pain was comparatively lower on days 2 and 3, table 5 .

Table 5 Postoperative Pain Score on the verbal rating scale (VRS)

\begin{tabular}{lllll}
\hline Pain Score & At Discharge $(\mathbf{n = 4 3 )}$ & $\begin{array}{l}\text { Day } \mathbf{1} \\
(\mathbf{n = 4 0 )}\end{array}$ & $\begin{array}{l}\text { Day 2 } \\
(\mathbf{n = 3 9 )}\end{array}$ & $\begin{array}{l}\text { Day 3 } \\
(\mathbf{n = 3 9 )}\end{array}$ \\
\hline No pain & $10(23.3)$ & $1(2.5)$ & $0(0)$ & $1(2.6)$ \\
Mild & $9(20.9)$ & $10(25.0)$ & $16(41.0)$ & $23(59.0)$ \\
Moderate & $10(23.3)$ & $15(37.5)$ & $11(28.2)$ & $11(28.2)$ \\
Severe & $14(32.6)$ & $14(35.0)$ & $12(30.8)$ & $4(10.3)$ \\
\hline \multicolumn{4}{r}{ Values in bracket are \% of total number }
\end{tabular}

The proportion of patients who experienced moderate to severe pain compared with No/mild pain was highest on day 1 , and the difference was statistically significant, $p$ value $=0.002$, table 6 .

Table 6 Difference of proportion between No/Mild Pain and Moderate/Severe Pain

\begin{tabular}{lllll}
\hline Postoperative Period & No/Mild & Moderate/Severe & X 2 & P-Value \\
\hline At Discharge & 19 & 24 & 0.581 & 0.446 \\
Day 1 & 11 & 29 & 10.000 & $0.002^{*}$ \\
Day 2 & 16 & 23 & 1.256 & 0.262 \\
Day 3 & 24 & 15 & 2.077 & 0.150 \\
\hline
\end{tabular}


A crosstabulation of factors associated with acute postoperative pain are illustrated in table 7.

Table 7 Factors affecting postoperative pain after $24 \mathrm{hrs}$

\begin{tabular}{lll}
\hline Category & No pain/Mild Pain & Moderate/Severe Pain \\
\hline $\begin{array}{l}\text { Types of Hernia } \\
\text { Unilateral }\end{array}$ & $9(25.7 \%)$ & $26(74.3)$ \\
$\begin{array}{l}\text { Bilateral } \\
\text { Cadre of Surgeon }\end{array}$ & $1(20.0)$ & $4(80.0)$ \\
Specialist & & \\
Medical Officer & $3(23.1)$ & $10(76.9)$ \\
Analgesic Regimen Used & $7(25.9)$ & $20(74.1)$ \\
Unimodal n(\%) & $2(20)$ & $17(56.7)$ \\
Multimodal n(\%) & $8(80)$ & $13(43.3)$ \\
Type of Anaesthesia & & \\
TIVA & $0(0.0)$ & $1(100.0)$ \\
LA & $7(36.8)$ & $12(63.2)$ \\
LA + TIVA & $0(0.0)$ & $7(100.0)$ \\
LA + Sedation & $2(16.7)$ & $10(83.3)$ \\
Spinal & $1(100.0)$ & $0(0.0)$ \\
\hline \multicolumn{2}{r}{ TIVA - Total Intravenous Anaesthesia, LA - Local Anaesthesia } \\
& Values in bracket are \% of total number
\end{tabular}

More patients who received unimodal analgesic regimen compared with multimodal regimen had moderate to severe pain (17 and 13 respectively).

\section{Discussion}

Surgical outreaches have been described as an important platform where the surgical disease burden in poor, rural African communities can be rapidly reduced [4, 8, 9]. In a recent study, Galukande et al., [4] reported that 536 surgical patients were treated within 4 days in 8 centres across Uganda. In this study, 148 surgical patients were treated, 58\% of whom had inguinal hernia. Hernias have been reported as the commonest surgical condition encountered during surgical outreaches in previous studies $[4,8,9]$. However, previous researchers have described their experience with inguinal hernia repairs during surgical camps $[4,8,9]$ few studies have prospectively investigated the morbidity, including acute pain associated with this important procedure in the immediate postoperative days $[10,11]$.

In this study, the incidence of moderate to severe pain was $55.8 \%, 72.5 \%, 59 \%$ and $38.5 \%$ on discharge, days 1,2 and 3 respectively. This is unacceptably high compared with previous studies on ambulatory herniorrhaphy $(5.5 \%)[10,11]$. The difference may be attributed to differences in study protocols. Apart from the physical and emotional suffering , poorly treated postoperative pain has been associated with increased incidence of nausea and vomiting, sleep disturbance, delayed ambulation, increased incidence of dangerous cardiopulmonary complications such as myocardial ischemia, stroke, deep vein thrombosis, pulmonary embolism, as well as progression to chronic pain which is often disabling and difficult to treat $[12,13]$.

Till present, there is no consensus among anaesthetists concerning the choice of anaesthesia for inguinal hernia repair, it varies from local, general and spinal anaesthesia $[8,9,14]$. However, local anaesthesia appears preferred in the setting of surgical outreaches where large volume of patients are treated, in that it can be administered by the surgeon, does not require recovery room facilities as the patient can go home immediately after surgery, with minimal side effects [14], nnevertheless, its contribution to postoperative pain varies [15]. In a previous randomized study, Tverskoy et al., [16] compared inhalational GA, GA plus incisional bupivacaine (GA Plus), and SA (spinal anaesthesia) for open hernia repair in 36 patients. They found that postoperative pain was significantly lower in GA plus group compared with GA and spinal only after 24 and 48 hours. In contrast, Teasdale et al., [17] reported no difference in pain after 6 and 24hours postoperatively when GA was compared with inguinal field block (IFB) using lidocaine in 103 patients undergoing herniorrhaphy. In our study (Table 2), 93\% of the procedures were performed under LA alone(44.2\%), or LA with either Total Intravenous Anaesthesia(18.6\%) or LA plus Sedation(30.2\%). General anesthesia (TIVA) and spinal anaesthesia 
(SA) were done in 2 and one patient respectively, hence group comparism will be erroneous. It is probable, the high incidence of moderate to severe pain observed in our study may have been due to the use of short acting lidocaine. Perhaps wound infiltration with long acting agents like bupivacaine or levobupivacaine would have improved the pain score.

A multimodal approach to postoperative pain management, in which two or more drugs acting on different points along the pain transmission pathway has been documented to be superior to any agent used alone, in terms of the quality of analgesia, reduction in opioid-related side effects [13].

In a previous study, Ausems et al., [10] showed that preventive and multimodal analgesia protocol improved pain score. In their institution, every adult ambulatory patient receives $1 \mathrm{~g}$ acetaminophen approximately 1 hour before surgery, $100 \mathrm{mg}$ diclofenac (if not contraindicated) upon arrival in the recovery room and, if indicated, opioid are titrated to achieve adequate postoperative pain control. At discharge from the day case centre, patients are instructed to use acetaminophen $1 \mathrm{~g}$ every 6hours and if needed diclofenac (50 mg three times daily). The addition of levobupivacaine wound infiltration to the above protocol further reduced the pain score. In our study, no standard protocol was followed. The prescription of analgesic medications was as per the surgeons' discretion. Only paracetamol and NSAIDS were prescribed. Opioid medications were not considered at all. The use of unimodal vis-à-vis multimodal analgesic was similar (46.5\% vs 53.5\%). Also the proportion of patients who experience moderate to serve pain were comparable between the two groups. (unimodal $56.7 \%$ Vs multimodal 43.3\%). This may be attributed to other factors. Majority of our patients did not take postoperative oral analgesics before discharge (69.8\%), this may have been due to the logistic challenge involved in obtaining the drugs from the central pharmacy, given the large crowd who queued up to receive their medications.

Also, the ward nurses did not give the prescribed oral analgesics even when they were available, neither was routine pain assessment done, rather they waited for the patients to complain of pain before they were given $75 \mathrm{mg}$ of intramuscular diclofenac. This practice may have been due to fear of giving oral medication within the immediate postoperative period.

The role of early administration of effective long acting analgesics before the effect of intraoperative analgesia and local anesthesia wears off, regular pain assessment as well as patient's education in improving acute pain management has been previously emphasized [13]. The development of a simple acute pain management protocol that is effectively communicated to all members of the surgical team including the ward nurses was lacking and it is advocated.

Before patients are discharged home from the ward, nurse-led discharge scoring systems such as post anesthetic discharge scoring system (PADSS), which include component of pain management should be applied to asses patients readiness for discharge [13]. In this study, all our patients were certified fit for discharge home by the doctors who were always very busy in the theatre leading to delayed discharge from the ward after full recovery.

Previous studies had reported that although many day case patients continue to experience pain and discomfort after discharge, 30-50\% do not take adequate analgesia due to misunderstanding and inadequate information [12, 13]. To improve compliance especially among patients with poor education, information on postoperative home pain relief should be conveyed both verbally and in written form [13]. In our study, only verbal information was provided, however drug compliance at home improved appreciably (Table 6), this may have been due to encouragement given by the researchers to the patients to take their medications during routine daily telephone interview in the study period.

Postoperative pain is often worse on the second postoperative day, when patients start to mobilize [13]. Therefore, all patients should be discharged home with adequate supply of analgesia to cover for breakthrough pain [13]. This agrees with our observation where activity was greater on the second postoperative day compared to day 1 (Table 7), however, the worse pain was experienced on day 1 (Table 8). It is possible some patients may have consulted local patent medicine dealers for more analgesics since this is a common practice in our environment.

Our study showed a high patients' satisfaction with their postoperative pain management at discharge $(69.8 \%$ in Table 9). This is rather ironic considering the high level of postoperative pain experienced (72.5\% for moderate/severe pain in Table 10). This seeming discrepancy between patient's satisfaction and postoperative pain was observed by previous researchers $[10,12]$. This is probably an expression of appreciation for the privilege of free surgical service and not necessarily adequacy of pain management. 
Finally, the small sample size used was a major limitation of our study. Therefore, a randomized study with an adequate sample size taking into consideration all the anaesthetic, surgical and analgesic factors affecting postoperative pain is indicated.

\section{Conclusion}

Surgical outreach program is useful in reducing surgical disease burden. However, the incidence of acute moderate to severe postoperative pain is high (72.5\%). Therefore, a standard analgesic protocol that is effectively communicated to all members of the surgical team including the ward nurses must be developed in order to reduce this morbidity.

\section{Compliance with ethical standards}

\section{Acknowledgments}

We sincerely appreciate Professor Sylvia G. Akpan for reading and correcting this work, and all the patients who gave their consent to participate in this study.

\section{Disclosure of conflict of interest}

The authors declare that there is no conflict of interest.

\section{Statement of ethical approval}

Approval for the study was obtained from the local Research Ethical Committees of the host medical facilities.

\section{Statement of informed consent}

All adult patients who underwent elective inguinal herniorrhaphy provided informed verbal consent to participate in the survey.

\section{References}

[1] Gosselin RA, Gyamfi Y and Contini S. (2011). Challenges of meeting surgical needs in the developing world. World J Surg, 35(2), 258-261.

[2] Contini S. (2007). Surgery in developing countries: why and how to meet surgical needs worldwide. Acta Biomed, 78, 4-5.

[3] Nordberg EM. (1984). Incidence and estimated need of caesarean section, inguinal hernia repair, and operation for strangulated hernia in rural Africa. Br Med J, 289(6437), 92-3.

[4] Galukande M, Kitueka O, Elobu E, Jombwe J, Sekabira J, Butter E and Faulac J. (2016). Improving surgical access in rural Africa through a surgical camp model. Surgery Research and practice.

[5] Hsia RY, Mbembati NA, Macfarlane S and Kruk ME. (2012). Access to emergency and surgical care in sub-saharan Africa: the infrastructure gap. Health Policy and Planning, 27(3), 234-244.

[6] Kalu QN, Eshiet AI, Ukpabio EI, Etiuma AU and Monjok E. (2004). A rapid need assessment survey of anaesthesia and surgical services in district public hospitals in Cross River State, Nigeria. BJMP, 7(4), a733.

[7] Onyekwulu FA, Nwosu ADG and Ajuzieogu VO. (2014). Anaesthesia manpower need in Nigeria. Orient Journal of Medicine, 26(3-4), 83-7.

[8] Ilori IU. (2012). Anaesthesia for surgical outreach in a rural Nigerian hospital. Afr J of Anaes Int Care, 12(1), 1620.

[9] Ojo E, Okoi E, Umoiyoho AJ and Nnamona M. (2013). Surgical outreach program in poor rural Nigerian communities. Rural Remote Health, 13(1), 2210.

[10] Ausems ME, Hulsewe KN, Hooymans PM and Hoofwijk AG. (2007). Postoperative analgesia requirement at home after inguinal hernia repair: effect of wound infiltration on postoperative pain. Anaesthesia, 62, 325-331. 
[11] Udo ID and Eyo CS. (2014). Day surgery: Are we transferring the burden of care? Niger J. Clinic practice, 17, 5025.

[12] McHugh GA and Thoms GMM. (2002). The management of pain following day-care surgery. Anaethesia, 57(3), 270-275.

[13] Tharakan L and Faber P. (2015). Pain management in day-case surgery. Continuing education in Anaethesia Critical Care \& Pain, (4), 180-183.

[14] Etta OE, Edubio MN and Nwalusi C. (2015). Anaesthesia for inguinal hernia repair: a review of practice at the University of Uyo Teaching Hospital, Uyo, Akwa Ibom State. W J Biomed Res, 2(1), 37-41.

[15] Callesen T and Kellet H. (1997). Postherniorhaphy pain. Anesthesiology, 87, 1219-1230.

[16] Tverskoy M, Cozacov C, Ayache M, Bradley EL and Kissin I. (1990). Postoperative pain after inguinal herniorhaphy with different types of anaesthesia. Anesth Analg, 70, 27-35.

[17] Teasdale C, McCrum A, William NB and Horton RE. (1982). A randomised controlled trial to compare local with general anaesthesia for short-stay inguinal hernia repair. Ann R Coll Surg Engl, 64, 238-42.

\section{How to cite this article}

Etta OE, Ekpe E, Inyang UC, Udeme N and Ekpenyong CE. (2020). Evaluation of acute pain management in ambulatory herniorrhaphy during free surgical outreach in Akwa Ibom State, South-South Nigeria. GSC Biological and Pharmaceutical Sciences, 11(2), 130-138. 